\title{
Chinese medicine Bazi Bushen capsule improves lipid metabolism in ovariectomized female $\mathrm{ApoE}^{-/-}$mice
}

\author{
Dan Huang ${ }^{1,2,3}$, Huawei $\mathrm{Hu}^{3}$, Liping Chang ${ }^{3}$, Shen Liu ${ }^{4}$, Junqing Liang ${ }^{3}$, Yanfei Song ${ }^{3}$, Xindong Wang ${ }^{1,2}$, \\ Hui Zhang ${ }^{5}$, Cong $\mathrm{Wei}^{3}$, Yiling $\mathrm{Wu}^{3}$ \\ ${ }^{1}$ Department of Cardiology, Affiliated Hospital of Integrated Traditional Chinese and Western Medicine, Nanjing University of Chinese Medicine, \\ Nanjing 210028, China; ${ }^{2}$ Department of Cardiology, Jiangsu Province Academy of Traditional Chinese Medicine, Nanjing 210028, China; ${ }^{3}$ National \\ Key Laboratory of Collateral Disease Research and Innovative Chinese Medicine, Shijiazhuang 050035, China; ${ }^{4}$ Department of Rehabilitation \\ Medicine, The First Affiliated Hospital of Shandong First Medical University, Jinan 250000, China; ${ }^{5}$ Department of Endocrinology, The First \\ Affiliated Hospital of Henan University of Chinese Medicine, Zhengzhou 450000, China \\ Contributions: (I) Conception and design: Y Wu, C Wei, D Huang; (II) Administrative support: Y Wu; (III) Provision of study materials or patients: \\ H Hu, L Chang, J Liang, Y Song; (IV) Collection and assembly of data: D Huang, S Liu, X Wang, H Zhang; (V) Data analysis and interpretation: D \\ Huang; (VI) Manuscript writing: All authors; (VII) Final approval of manuscript: All authors. \\ Correspondence to: Cong Wei; Yiling Wu. National Key Laboratory of Collateral Disease Research and Innovative Chinese Medicine, Shijiazhuang \\ 050035, China. Email: weicong@yiling.cn; wuyiling68@126.com.
}

Background: Dyslipidemia is one of the mechanisms of atherosclerosis (AS). Depletion of estrogen plays a key role in the pathogenesis of postmenopausal AS in women, and the blood lipid levels of women are closely related to endogenous estrogen levels. Phytoestrogens (PEs) exert estrogenic effects, including protection against AS, without the adverse effects of estrogen administration. Bazi Bushen capsule (BZBS) is a traditional Chinese medicine herbal compound prescription that has been shown to contain 11 unique PEs. In the present study, we assessed the effects of BZBS against lipid metabolism disorders.

Methods: All ApoE ${ }^{-/-}$mice underwent ovary ligation and bilateral ovariectomy (Ovx) to induce surgical menopause (Ovx/ApoE ${ }^{-/-}$mice), whereas the C57BL/6J mice underwent sham surgery (needle threading). $\mathrm{Ovx} / \mathrm{ApoE}^{-/-}$mice were given a high-fat diet without estrogen and C57BL/6J mice were given a normal diet for 12 weeks. Ovx/ApoE ${ }^{-/-}$mice treated with G1, a highly selective G-protein-coupled estrogen receptor1 (GPER1) agonist with proven activity against AS, were used as positive controls. Estrogen levels were measured and uterine atrophy index was calculated to determine the success of the model. Serum levels of triglycerides (TG), total cholesterol (TC), low-density lipoprotein cholesterol (LDL-C) and high-density lipoprotein cholesterol (HDL-C) were measured in each group. The orthogonal projections to latent structures discriminant analysis (OPLS-DA) model was used to separate the groups, MetaboAnalyst was then used to analyze the metabolic pathway, and the most representative metabolites were finally identified.

Results: Removal of bilateral ovaries resulted in successful surgical menopause models, where BZBS increased estrogen levels but did not increase the risk of uterine proliferation. BZBS attenuated dyslipidemia, including decreased TG, TC, and LDL-C levels, but increased HDL-C levels. The OPLS-DA model successfully distinguished the groups with good predictive ability and revealed their tendency to separate from each other. MetaboAnalyst suggested that both the G1 group and high-dose BZBS (HD-BZ) could improve the effect of lipid metabolism: the glycerophospholipid metabolism pathway was mainly improved by the G1 group, while the inositol phosphate metabolism pathway was mainly improved by the HD-BZ group. For the four compounds with the highest content, the concentrations of docosahexaenoic acid (DHA), 3-hydroxybutyric acid, and 5(Z), 8(Z), 11(Z)-eicosatrienoic acid were dramatically lower in the model group compared to the control group. Lysophosphatidylethanolamine (18:0) was higher in the model group than in the control group. BZBS corrected these effects.

Conclusions: BZBS treatment reduced serum lipid levels and improved fatty acid metabolism in high-fat diet-fed, surgically induced menopausal $\mathrm{ApoE}^{-/-}$mice. 
Keywords: Atherosclerosis (AS); Bazi Bushen capsule; lipid profile; metabonomics

Submitted Mar 25, 2020. Accepted for publication Apr 28, 2020.

doi: 10.21037/apm-20-906

View this article at: http://dx.doi.org/10.21037/apm-20-906

\section{Introduction}

Atherosclerosis (AS), the common underlying pathology of cerebral and cardiovascular diseases, is the leading cause of death worldwide $(1,2)$. The risk of AS in premenopausal women is much lower than that in men; however, the risk increases significantly after menopause. These differences suggest that estrogen, a steroid hormone that performs various physiological functions by binding to its corresponding receptors, plays a key protective role in the pathogenesis of AS (3). Dyslipidemia is one of the mechanisms of AS. The blood lipid levels of women are closely related to endogenous estrogen levels (4). Estrogen is synthesized in the ovaries using low-density lipoprotein cholesterol (LDL-C) as a substrate. However, LDL-C in the blood of postmenopausal women cannot be used to synthesize estrogen, leading to estrogen deficiency and further dyslipidemia (5). The accumulation of subcutaneous and visceral fat in postmenopausal women is more obvious than that before menopause, leading to abnormal fatty acid metabolism. The decomposition of triglycerides (TG) into glycerol and free fatty acids increases, which increased lipid deposition (6).

Despite the potent vascular protective activity of estrogen, its clinical application has been limited by several potential side effects, such as headaches, thrombosis, endometrial hyperplasia with high cancer risk, breast pain, and other disorders (7). Phytoestrogens (PEs), phenolicring-containing molecules similar in structure to estrogen, can bind to estrogen receptors. They exert protective estrogenic activity against AS without the adverse effects caused by estrogen administration (7). Bazi Bushen capsule (BZBS; Eight-seed Kidney-Tonifying Capsule) is a herbal compound used in traditional Chinese medicine. It is derived from Wuzi Yanzong Wan (Five-Seed Progeny Pill), which is another popular prescription, famously recorded by a doctor named Li Chan (1518-1593) during the Ming Dynasty in his book Yi Xue Ru Men (Introduction to Medicine). We identified 14 components in BZBS, 11 of which were PEs including epimedin A, icariin, baohuoside I, isoquercitrin, hyperoside, osthole, imperatorin, deoxyschizandrin, schisandrin B, catalpol, and verbascoside.

For the present study, we aimed to assess the potential use of BZBS against lipid metabolism. To this end, we examined the effects of BZBS oral gavages on blood lipid and serum metabolomics in ovariectomized (Ovx) $\mathrm{ApoE}^{-/-}$ mice, an animal model of post-menopausal AS. G1, a selective G-protein-coupled estrogen receptor1 (GPER1) agonist that has already been shown to decrease blood lipid level and hinder AS in post-menopausal conditions, was used as a positive control. Our results revealed that BZBS reduced serum lipid levels and improved lipid metabolism in $\mathrm{Ovx} / \mathrm{ApoE}^{-/-}$mice by affecting fatty acid metabolism pathways.

We present the following article in accordance with the ARRIVE reporting checklist (available at http://dx.doi. org/10.21037/apm-20-906).

\section{Methods}

\section{Preparation and quality control (QC) of BZBS}

BZBS samples were obtained from Shijiazhuang Yiling Pharmaceutical Co., Ltd (Shijiazhuang, China). BZBS consists of 14 components. In the process of BZBS production, the species, origin, harvest time, medicinal parts, and concocted methods for each component were strictly standardized according to the Chinese Pharmacopoeia (2015 Edition). The stability of the product was verified by ultra-performance liquid chromatography (UPLC) analysis of 12 batches of BZBS. The respective chromatographs and the 14 identified "fingerprints" are shown in Figure $1 A$ and $B$. BZBS was administered as an intragastric injection into mice in a suspension of $0.5 \%$ sodium carboxymethyl cellulose (CMC).

\section{Preparation of $G 1$}

G1 was purchased from ApexBio (Houston, TX, USA). A stock solution was prepared by dissolving G1 in absolute ethanol at $20 \mu \mathrm{g} / \mathrm{mL}$. The working solution $(2 \mu \mathrm{g} / \mathrm{mL})$ was prepared by diluting the stock in 9 volumes of the aqueous vehicle $(0.9 \% \mathrm{NaCl}, 0.1 \%$ albumin, and $0.1 \%$ Tween- 20$)$. Mice were injected subcutaneously with $100 \mu \mathrm{L}$ of the working solution each day (8). 

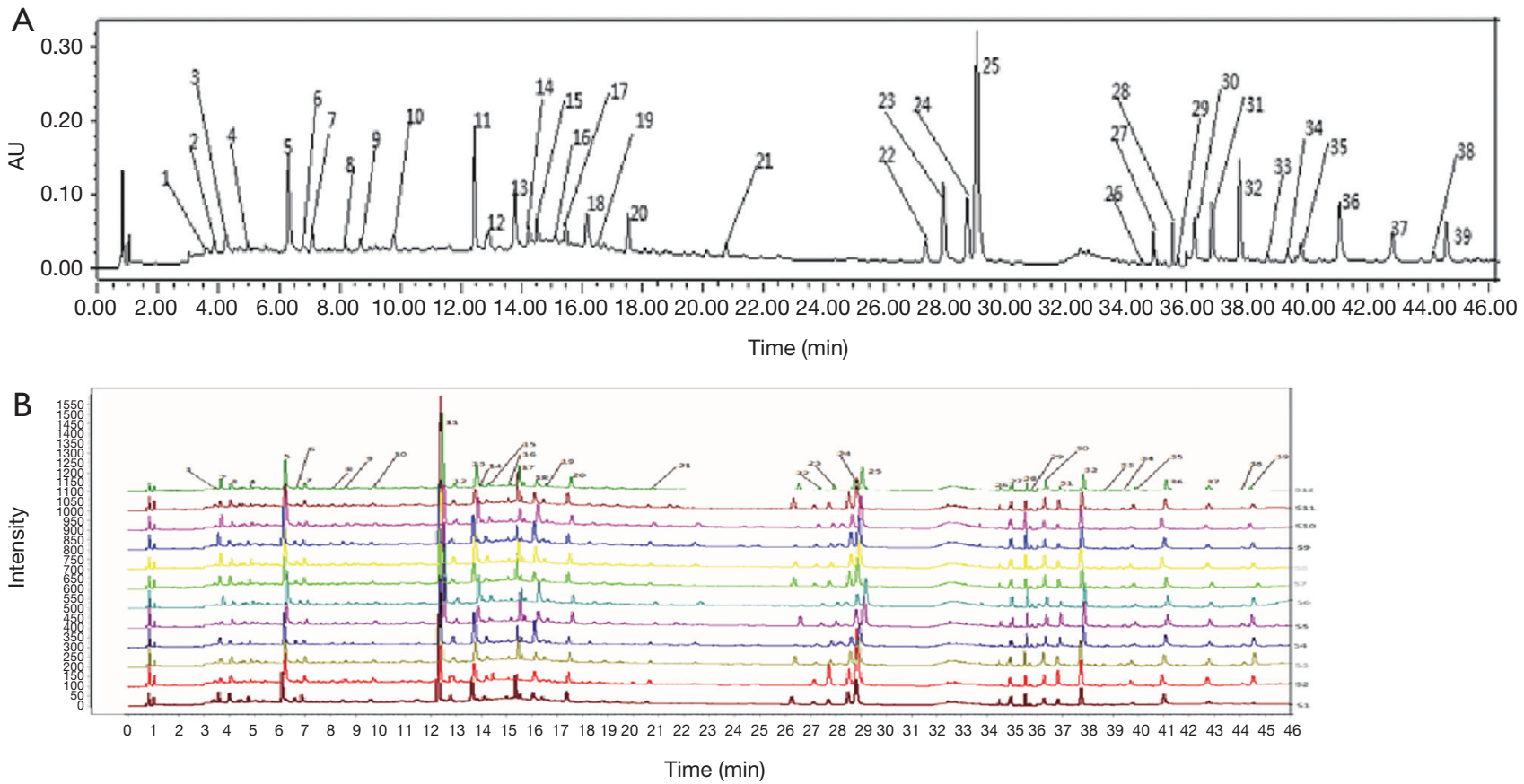

Figure 1 Chromatographic fingerprints of BZBS. (A) Representative UPLC chromatograph of BZBS fingerprints. The peaks are as follows: 3, neochlorogenic acid; 5, chlorogenic acid; 7, cryptochlorogenic acid; 13, isoquercitrin; 14, hyperoside; 17, verbascoside; 22, epimedin A; 24, icariin; 29, baohuoside I; 31, imperatorin; 32, osthole; 36, catalpol; 37, deoxyschizandrin; 39, schisandrin B. (B) Chromatographic fingerprints obtained from 12 BZBS batches (S1-S12:BZ1-BZ12).

\section{Grouping and drug administration}

Sixty female homozygous Apo: $\mathrm{E}^{-/-}$mice (of C57BL/6J background) and fifteen female C57BL/6J control mice (6-8 weeks old and weighing 18-22 g) were purchased from GemPharmatech Co., Ltd. (Nanjing, China). All mice were housed in a standard facility at $22{ }^{\circ} \mathrm{C}$ under a $12 \mathrm{~h}$ lightdark cycle with food and water available ad libitum. After a 3-day adaptation period, all $\mathrm{ApoE}^{-/-}$mice underwent ovary ligation and bilateral ovariectomy $(\mathrm{Ovx})$ under sterile conditions to induce surgical menopause $\left(\mathrm{Ovx} / \mathrm{ApoE}^{-/-}\right.$ mice), whereas the $\mathrm{C} 57 \mathrm{BL} / 6 \mathrm{~J}$ mice underwent sham surgery (needle threading). Animals were left to recover for 7 days to ensure that the sex hormones in their bodies were eliminated. Afterward, the $\mathrm{Ovx} / \mathrm{ApoE}^{-/-}$mice were randomly assigned into four groups ( $\mathrm{n}=15$ per group): the model group (high-fat diet + CMC via intragastric injection), the G1 treatment group (high-fat diet + G1 $0.2 \mu \mathrm{g} /$ day via subcutaneous injection), the high-dose BZBS (HD-BZ) group (high-fat diet $+2.8 \mathrm{~g} / \mathrm{kg} /$ day BZBS via intragastric injection), and the low-dose BZBS (LD-BZ) group (high-fat diet $+1.4 \mathrm{~g} / \mathrm{kg} /$ day BZBS via intragastric injection). The $15 \mathrm{C} 57 \mathrm{BL} / 6 \mathrm{~J}$ mice were used as the control group (normal diet + CMC via intragastric injection).

Normal diet (standard commercial diet) was purchased from Moldiets Co., Ltd (Chengdu, China). High-fat diet (PE-free, $40 \mathrm{kcal} \%$ fat, $1.25 \%$ cholesterol, and $0.5 \%$ cholic acid) was purchased from Research Diets, Inc (New Brunswick, NJ, USA).

\section{Uterus atrophy index (mg/g) determination}

After 12 weeks on normal or high-fat diet, all mice were fasted overnight and weighed for the last time. The animals were sacrificed and each uterus was taken and weighed. For each mouse, a uterus atrophy index was determined, which was defined as the weight of the uterus (in $\mathrm{mg}$ ) divided by the total body weight (in $\mathrm{g}$ ).

\section{Serum estradiol and lipid levels analyses}

The serum of mice from each group were separated by centrifugation at 3,000 $\mathrm{rpm}$ for $15 \mathrm{~min}$ and collected. Estradiol (E2) measurements were performed by Beijing North Institute of Biotechnology Co., Ltd. (Beijing, China). 

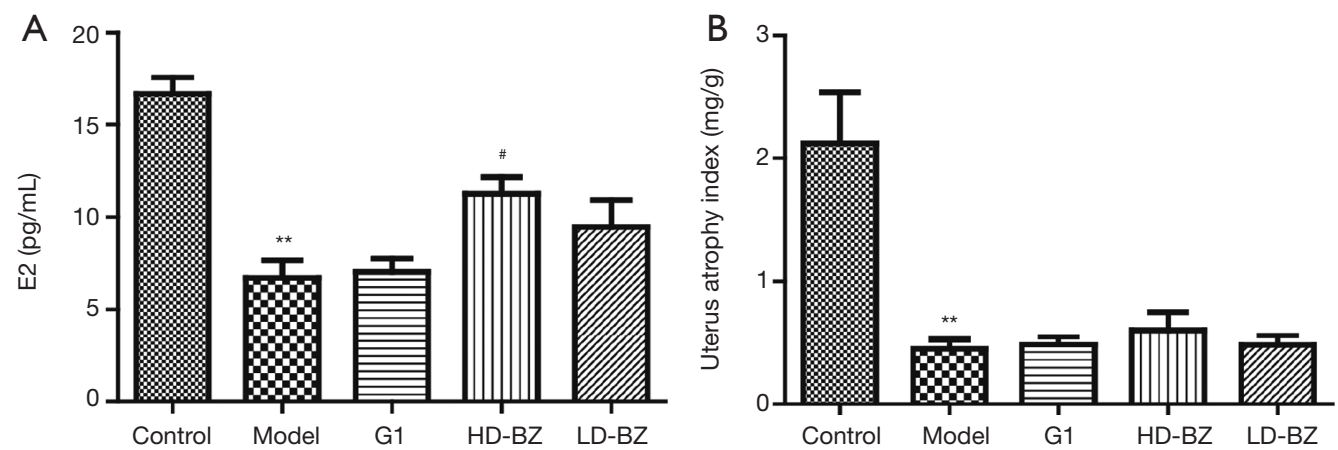

Figure 2 Effects of BZBS on the estrogen levels (A) or uterus atrophy index values (B) of high fat diet-induced atherosclerotic Ovx/ApoE ${ }^{-/}$ mice at 12 weeks. ${ }^{* *}, \mathrm{P}<0.01$ vs. the control group; ${ }^{\prime}, \mathrm{P}<0.05$, vs. the model group $(\mathrm{n}=6-15)$.

Total cholesterol (TC), TG, and LDL-C biochemical kits were purchased from Beijing Strong Biotechnologies, Inc. (Beijing, China). The lipid levels were detected by automatic biochemical analyzer (Hitachi 7080, Japan).

\section{Sample extraction and ultra-performance liquid chromatography-mass spectrometry (UPLC-MS) analysis}

The serum from the mice was processed to undergo ultraperformance liquid chromatography-mass spectrometry (UPLS-MS) analysis. Extraction and analysis were performed as described previously with a few modifications (9). In brief, $50 \mu \mathrm{L}$ of serum was added to a tube containing $200 \mu \mathrm{L}$ of a mixed precipitator (methanol: acetonitrile at 5:3, v/v) to extract metabolites and precipitate proteins. A $20 \mu \mathrm{L}$ aliquot of each supernatant was mixed for performance QC samples. Serum metabolites were separated using an ACQUITY UPLC HSS T3 column $(2.1 \mu \mathrm{L} \times 150 \mathrm{~mm} \times$ $1.8 \mathrm{~mm}$ ) (Waters Corp., Milford, MA, USA) at $40{ }^{\circ} \mathrm{C}$ and at a flow rate of $0.35 \mathrm{~mL} / \mathrm{min}$. The injection volume was $3 \mu \mathrm{L}$; the two mobile phases and separation have been described previously (9). An AB5600 mass spectrometer equipped with electron spray ionization (ESI) source was used to acquire mass spectral profiles (AB Sciex LLC, Redwood City, CA, USA). The optimized operating parameters were as follows: source voltage, $5.50 \mathrm{kV}$ (positive mode) and $4.50 \mathrm{kV}$ (negative mode); mass spectra were acquired by scanning over the $50-1,200 \mathrm{~m} / \mathrm{z}$ range.

\section{Statistical analysis}

Orthogonal projection to latent structures discriminant analysis (OPLS-DA) was performed with SIMCA-P 13.0 software (Umetrics AB, Umeå, Sweden) based on the dataset. Statistical analysis was done by one-way analysis of variance (ANOVA) if more than two groups were compared. Correlation analysis was performed using SPSS 17.0 software (SPSS Inc., Chicago, IL, USA). Differences with $\mathrm{P}$ value $<0.05$ were considered statistically significant. All results were presented as mean \pm standard error of the mean.

\section{Results}

\section{BZBS treatment increased serum estradiol levels without uterine proliferation}

Compared with the control group, the E2 levels of the model group were substantially lower, confirming that the bilateral ovariectomy resulted in a post-menopause-like state. There was no significant change in E2 levels between the G1 group and the model group. In contrast, the concentration of E2 was elevated in both the HD-BZ and the LD-BZ group (Figure 2A).

With respect to uterus atrophy, the index values of all $\mathrm{Ovx} / \mathrm{ApoE}^{-/-}$mice were significantly lower than those of the control group (C57BL/6 mice). In other words, the G1 group and BZBS groups had no obvious effect on uterine nutrition (Figure 2B).

\section{BZBS treatment improved the serum lipid levels in Ovx/ ApoE $^{-/-}$mice}

Oxv $\mathrm{ApoE}^{-/-}$mice were fed an atherogenic diet for 12 weeks. At the end of this period, serum lipid levels were assessed in order to evaluate whether BZBS treatment can improve serum lipid levels. The serum levels of all lipids were significantly higher in the model group than in the control 

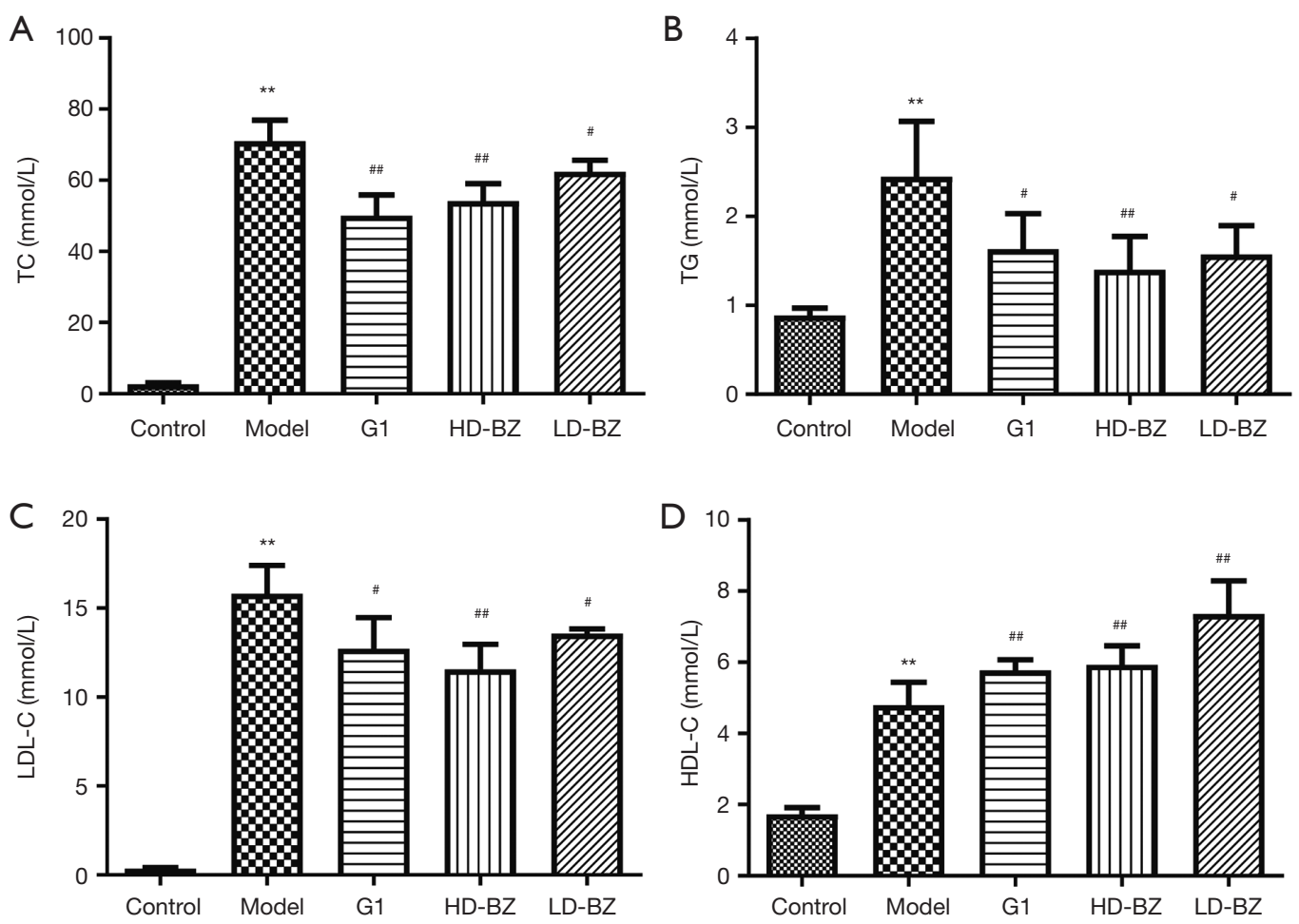

Figure 3 Effects of BZBS on serum lipids of high fat diet-induced atherosclerotic Ovx/ApoE ${ }^{-/-}$mice at 12 weeks. ${ }^{* *}$, $\mathrm{P}<0.01$ vs. the control group; $, \mathrm{P}<0.05,{ }^{\#}, \mathrm{P}<0.01$ vs. the model group (n=10-15).

group. In the case of TC, TG, and LDL-C, treatment with G1 or BZBS statistically significantly decreased these levels compared to the model group, with high-dose BZBS having a stronger effect than low-dose BZBS. In contrast, G1 and BZBS treatment actually increased the HDL-C level even higher, but the effect was greater in the low-dose BZBS group (Figure 3).

\section{BZBS treatment had a positive effect on metabolic shifts}

After preprocessing, 1,358 ion signals (845 in positive mode and 513 in negative mode) were detected in the raw data and aligned in our high-resolution accurate mass UPLC-MS platform. Our OPLS-DA model successfully distinguished the groups with good predictive ability and revealed their tendency to separate from each other. The model group was the farthest from the control group. The G1 group migrated towards the control group, while the position of the high- and low-dose BZBS groups was similar to that of the G1 group. The high-dose group was slightly closer to the control group than the low-dose group, suggesting BZBS had a dose-dependent effect (Figure 4).

\section{BZBS treatment affects fatty acid metabolic patbways}

MetaboAnalyst was used to analyze the metabolic pathway. The main differences between the model group and the control group were found to be the glycerophospholipid metabolism and the inositol phosphate metabolism pathway (Figure $5 A$ ). Figure $5 B$ shows that the difference between G1 and the model group was the improvement of 2 pathways, namely, glycerophospholipid metabolism and inositol phosphate metabolism. According to Figure 5C, the difference between G1 and the normal control group was mainly concentrated on inositol phosphate metabolism; in other words, it almost completely improved the glycerophospholipid metabolism pathway. Meanwhile, Figure $5 D$ illustrates the difference between the HDBZ group and the model group. The HD-BZ group also improved the two pathways of glycerophospholipid metabolism and inositol phosphate metabolism. Figure $5 E$ shows the difference between the HD-BZ group and the normal group. Unlike G1, the HD-BZ group restored the inositol phosphate metabolism pathway to a normal level. The results suggest that both G1 and HD- 


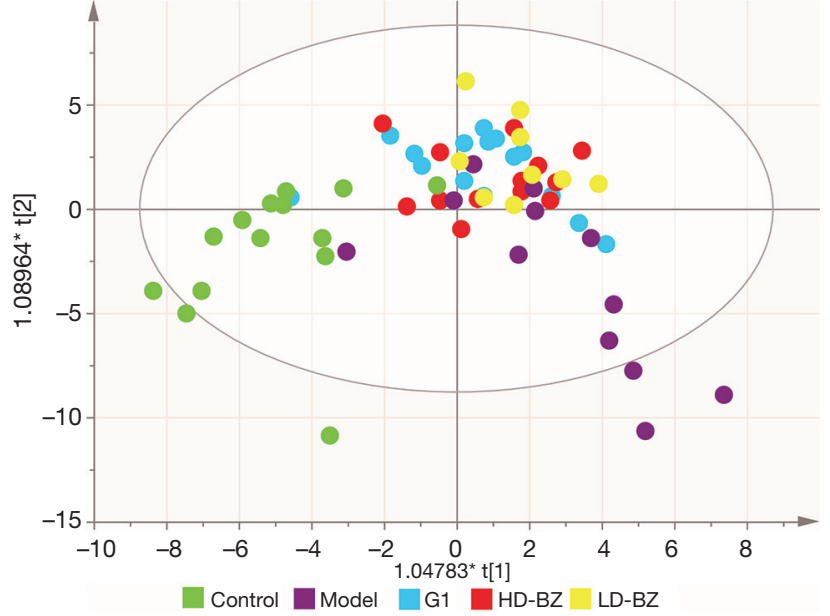

Figure 4 OPLS-DA modeling demonstrates the effects of BZBS on metabolic shifts in high-fat diet-induced atherosclerotic Ovx/ $\mathrm{ApoE}^{-/-}$mice at 12 weeks. $(\mathrm{n}=9-15)$.
BZ could improve the effect of lipid metabolism: the glycerophospholipid metabolism pathway was mainly improved by in the G1 group, while the inositol phosphate metabolism pathway was mainly improved in the HD-BZ group.

\section{BZBS treatment improved the metabolism of fatty acid- related differential compounds}

For the 4 compounds with the highest content, the concentrations of docosahexaenoic acid (DHA); 3-hydroxybutyric acid; and $5(Z), 8(Z), 11(Z)$-eicosatrienoic acid were dramatically lower in the model group compared to the control group. G1 and BZBS tended to alleviate this effect, as they restored the levels to normal. In contrast, the lysophosphatidylethanolamine (18:0) level was higher in the model group than in the control group. G1 and BZBS again
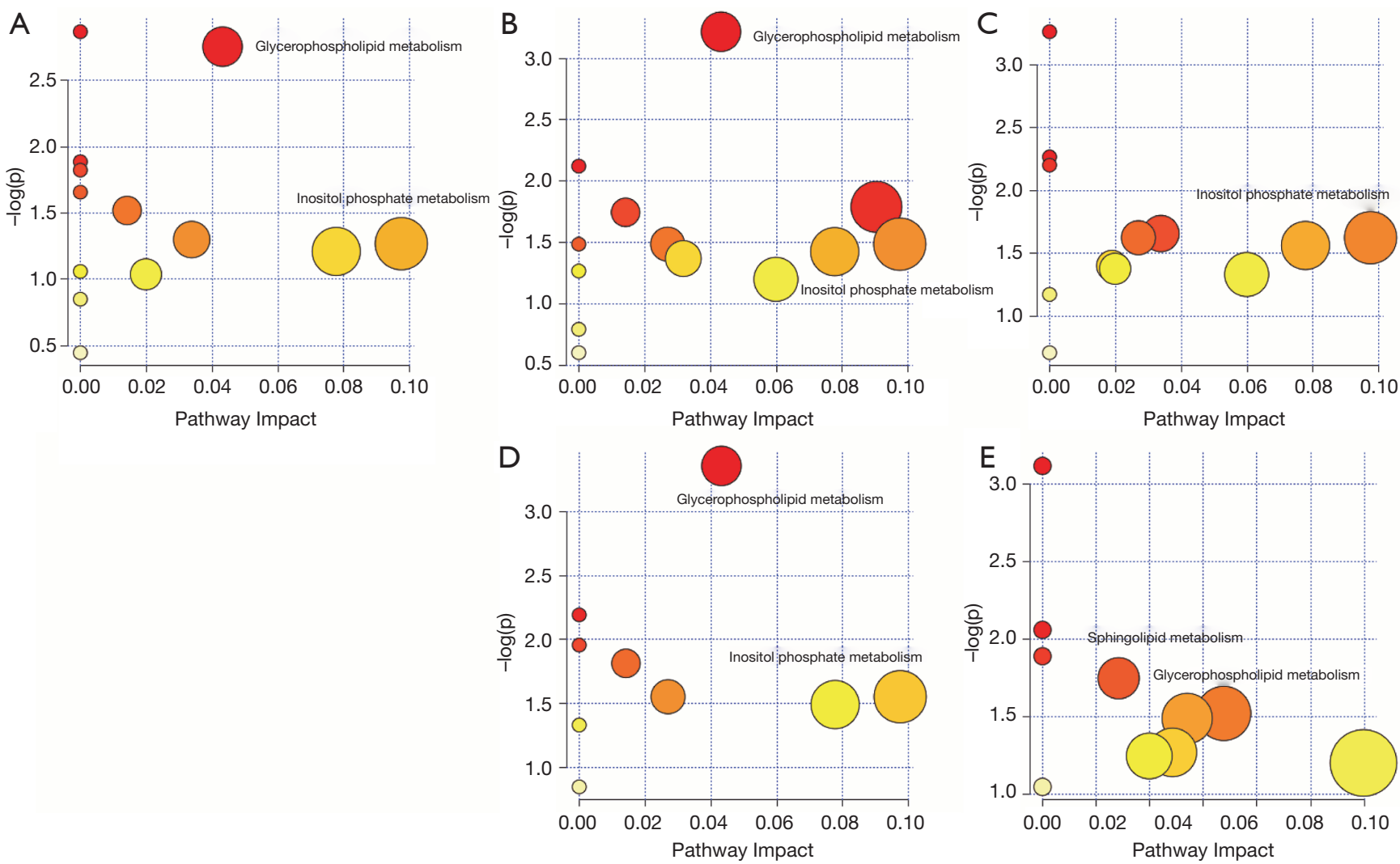

Figure 5 Metabolic pathway analysis diagram in high-fat diet-induced atherosclerotic Ovx/ApoE ${ }^{-/}$mice serum. (A) Model group $v s$. control group; (B) G1 group vs. model group; (C) G1 group vs. control group; (D) HD-BZ group vs. model group; (E) HD-BZ group vs. control group. $(\mathrm{n}=9-15)$. 

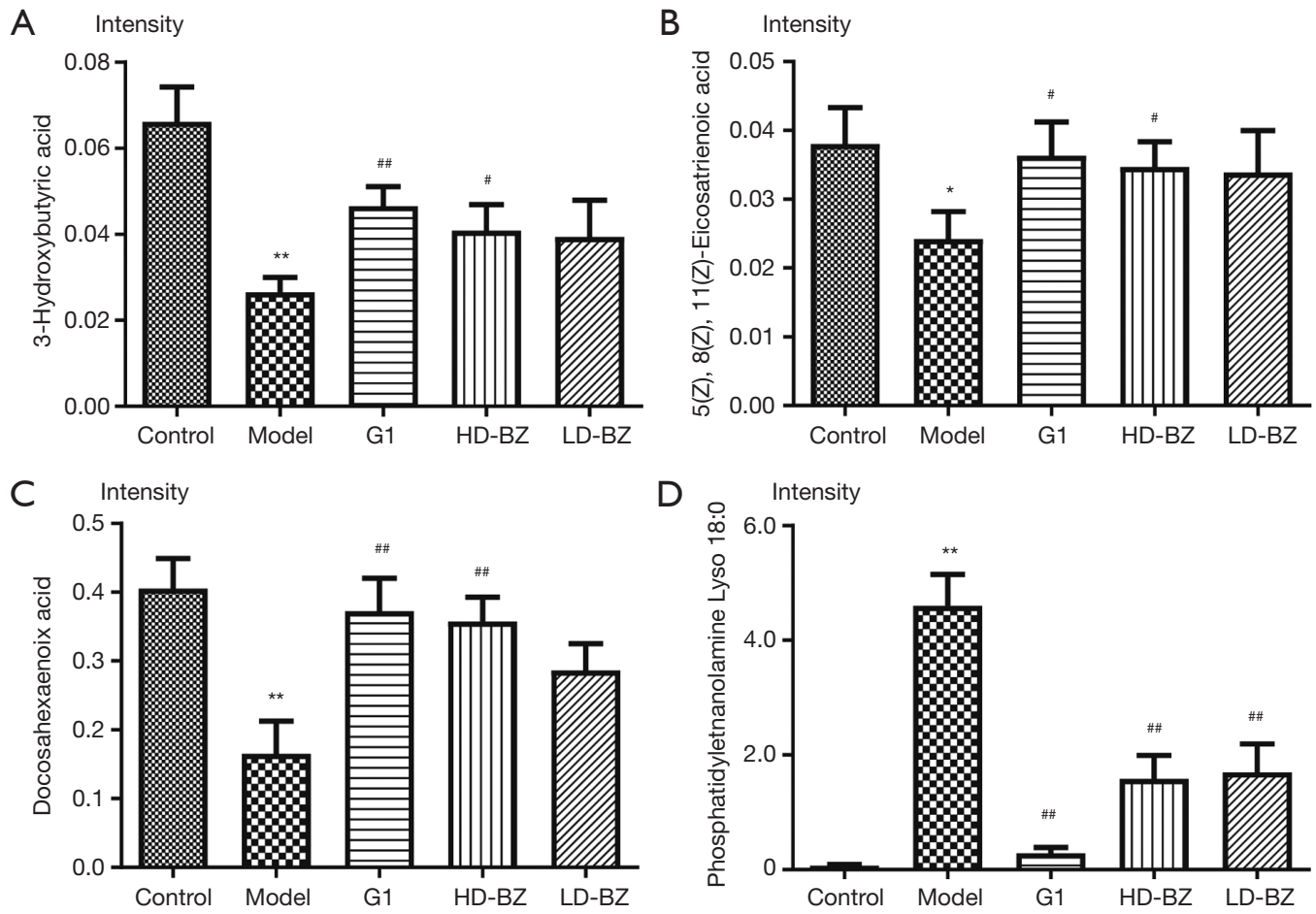

Figure 6 Effect of drugs on metabolite levels in high-fat diet-induced atherosclerotic Ovx/ApoE ${ }^{-/-}$mice serum. (A) Docosahexaenoic acid; (B) 3-hydroxybutyric acid; (C) 5(Z), 8(Z), 11(Z)-eicosatrienoic acid; (D) lysophosphatidylethanolamine $(18: 0)$. (n=9-15). *, $\mathrm{P}<0.05,{ }^{* *}, \mathrm{P}<0.01$ vs. the control group; ${ }^{\#}, \mathrm{P}<0.05,{ }^{\#}, \mathrm{P}<0.01$ vs. the model group.

alleviated the effect through decreasing the concentration to levels closer to that of the control group (Figure $6 A, B, C, D$ ). These results indicate that both the metabolome and endogenous small molecules demonstrated the effect of BZBS.

\section{Discussion}

Estrogen has three receptors: the classic nuclear receptor (ER $\alpha$ and ER $\beta$ ) and the membrane receptor GPER1 (10). GPER1 agonist G1 was selected as a positive control because G1 is devoid of uterotrophic activity, which is similar to PEs (11). Our study demonstrated that BZBS did not prevent uterus atrophy in $\mathrm{Ovx} / \mathrm{ApoE}^{-/-}$mice (Figure $2 B$ ). It is known that after the cessation of estrogen production by the ovaries, E2 biosynthesis takes place in peripheral tissues, especially adipose tissue, through aromatase conversion of androgens in postmenopausal women (12). Several studies have shown that PEs elevate the levels of E2 in female mice, and BZBS can also increase serum E2 levels $(13,14)$. This is likely because PEs can competitively bind to ER $\alpha$ and ER $\beta$, and the amount of ER in plasma is limited. Endogenous estrogen cannot bind to the receptor and will be unbound in the plasma, leading to an increase in E2 plasma levels $(13,15)$. As a traditional Chinese medicine, BZBS is composed of a complex molecular mixture and has multi-target effects. Thus, one cannot rule out that ER $\alpha$ and ER $\beta$ play a role in BZBS activity.

AS is characterized by the accumulation of lipids in the artery wall $(16,17)$, and dyslipidemia is a major risk factor for the development of AS $(18,19)$. Previous research has demonstrated that PEs may prevent the progression of atherosclerotic lesions by improving lipid metabolism (20), and the present study additionally found that BZBS significantly lowered the serum levels of TC, TG, and LDL-C, while raising that of HDL-C. The role of BZBS in regulating blood lipids was related to its active components. Isoquercitrin has been demonstrated to enhance AMPK phosphorylation, up-regulate the expression of AdipoR1, and down-regulate the expression of sterol regulatory element-binding protein transcription factor 1 (SREBP-1) and fatty acid synthase (FAS) genes to regulate lipid accumulation (21). Meanwhile, hyperoside has been shown to inhibit fat formation by inhibiting the expression of 
transcription factors and fat-forming genes and reducing lipid accumulation in 3T3-L1 adipocytes (22). Epimedin A and icariin inhibited HDL oxidation and glycation, mainly by inhibiting peroxidation of lipids, accumulation of apoA1 , increase of negative charge, and formation of advanced glycosylation end products (AGEs). Also, osthole and catalpol have been observed to reduce the levels of TC, TG, and LDL-C in the blood of high-fat fed mice or rats, while increasing the levels of HDL-C (23). The possible mechanism by which osthole regulates lipid metabolism is to inhibit liver lipid accumulation through activation of AMPK pathway, improve lipolysis, and inhibit the expression of adipogenesis transcription factors (24). Imperatorin downregulated the important transcription factors peroxisome proliferator-activated Receptor $\gamma$ (PPAR- $\gamma$ ) and SREBP-1 for lipid synthesis (25). Schisandrin B increased protein kinase A (PKA)-mediated hormone sensitive lipase (HSL) activity, increased lipolysis and fatty acid oxidation gene expressions, thereby regulating lipid metabolism (26). However, the effect of BZBS on HDL-C that we observed is inconsistent with previous research results. Many studies report that PEs dose-dependently affect HDL-C; that is, high-dose PEs increased HDL-C level more significantly, while BZBS was the lower-dose group that elevated HDL-C better $(27,28)$. In fact, the effect of estrogen on HDL-C is controversial, and other studies have shown that estrogen does not regulate HDL-C $(29,30)$. Further research is necessary to explore the complex relationship of estrogen and PEs.

The metabolic pathway analysis showed that G1 and BZBS improved both glycerophospholipid metabolism and inositol phosphate metabolism, and we further identified the metabolites. The metabolomics analysis identified DHA; 3-hydroxybutyric acid, 5(Z), 8(Z); 11(Z)-eicosatrienoic acid; and lysophosphatidylethanolamine (18:0), as the four most abundant differentially expressed compounds. Both of the differential compounds and both of the metabolic pathways are related to fatty acid metabolism (31). Vascular endothelial inflammation and apoptosis are important mechanisms for the development of AS (32). DHA is an n-3 polyunsaturated fatty acid (PUFA) that has been demonstrated to inhibit atherogenesis (33), possibly by reducing endothelial cell inflammation (34). DHA also reduces endothelial apoptosis by decreasing transcription of the anti-apoptotic gene cIAP1, which is involved in gene coding for an endogenous caspase inhibitor that reduces caspase-3 activity (35). $\beta$-Hydroxybutyrate $(\beta-\mathrm{HB})$ is a fatty acid-derived molecule mainly produced in the liver. $\beta$-HB is transported by blood to peripheral tissues (including the brain, heart, and skeletal muscles), where it provides the energy required by tissues during fasting (36). $\beta$-HB is not only a metabolic intermediate but also possesses a variety of signaling functions (37). It is known to downregulate caspase- 3 and to significantly increase the expression of Bcl-2 (38,39). Epoxyeicosatrienoic acids (EETs) are biologically active epoxide derivatives products of arachidonic acid that are formed by cytochrome P450 (CYP) epoxygenases, and they exhibit potent antiinflammatory properties and vascular-protecting effects $(40,41)$. EET regioisomers differ in their anti-inflammatory effects, with 11 and 12-EET being the most effective (42). EETs have found to be able to reduce the expression of adhesive molecules VCAM-1, ICAM-1, and E-selectin in cytokine-induced endothelial cells (ECs) through the I $\kappa \mathrm{B}$ kinase/NF- $\mathrm{BB}$ pathway (42). The anti-apoptotic effects of EETs have been associated with inhibition of the Bcl-2/Bax/ caspase 3 signaling pathway (43). Phospholipids are the main structural components of cell membranes and contribute greatly to membrane biochemical and biophysical properties. Phosphatidylethanolamine, phosphatidylcholine, and phosphatidylserine are major phospholipids of the membrane of mammalian cells (44). Phosphatidylethanolamine has been shown to suppress the activity of MAPK (45) and Raf-1 kinase (46), while activating heterotrimeric G proteins (47). Lysophosphatidylethanolamine (LPE) is a phosphatidylethanolamine molecule lacking a fatty acid chain (48). Accumulating evidence suggests that cell apoptosis has a pivotal effect on membrane lipid homeostasis, causing membrane destruction or cell structure collapse (49). Our study was consistent with previous studies that reported fatty acid metabolites being significantly higher in postmenopausal women than in normal women (50), with BZBS correcting for this effect.

\section{Conclusions}

Our study demonstrated that BZBS treatment reduced serum lipid levels and improved fatty acid metabolism in high-fat diet-fed, surgically induced menopausal $\mathrm{ApoE}^{-/-}$ mice. Our findings indicate that BZBS may be a potential pharmaceutical agent for the prevention of AS in postmenopausal women.

\section{Acknowledgments}

Funding: This work was supported by the National Key Research and Development Program-Modernization 
of Traditional Chinese Medicine (grant number 2017YFC1700501) and the National Natural Science Foundation of China (grant number 81973766).

\section{Footnote}

Reporting Checklist: The authors have completed the ARRIVE reporting checklist. Available at http://dx.doi. org/10.21037/apm-20-906

Data Sharing Statement: Available at http://dx.doi. org/10.21037/apm-20-906

Conflicts of Interest: All authors have completed the ICMJE uniform disclosure form (available at http://dx.doi. org/10.21037/apm-20-906). All authors report grants from China National Center for Biotechnology Development, grants from National Natural Science Foundation of China, during the conduct of the study.

Ethical Statement: The authors are accountable for all aspects of the work in ensuring that questions related to the accuracy or integrity of any part of the work are appropriately investigated and resolved. All animal experiments were executed according to the Guidelines and Policies for Animal Surgery of the Hebei Yiling Chinese Medicine Research Institute (Shijiazhuang, China) and approved by the Ethical Commission of Animal Care under the approval number N2018050 (20180711).

Open Access Statement: This is an Open Access article distributed in accordance with the Creative Commons Attribution-NonCommercial-NoDerivs 4.0 International License (CC BY-NC-ND 4.0), which permits the noncommercial replication and distribution of the article with the strict proviso that no changes or edits are made and the original work is properly cited (including links to both the formal publication through the relevant DOI and the license). See: https://creativecommons.org/licenses/by-nc-nd/4.0/.

\section{References}

1. Geraci G, Mule G, Mogavero M, et al. Renal haemodynamics and severity of carotid atherosclerosis in hypertensive patients with and without impaired renal function. Nutr Metab Cardiovasc Dis 2015;25:160-6.

2. Sima $\mathrm{P}$, Vannucci L, Vetvicka V. Atherosclerosis as autoimmune disease. Ann Transl Med 2018;6:116.
3. Rexrode K. Sex Differences in Sex Hormones, Carotid Atherosclerosis, and Stroke. Circ Res 2018;122:17-9.

4. Marchand GB, Carreau AM, Weisnagel SJ, et al. Increased body fat mass explains the positive association between circulating estradiol and insulin resistance in postmenopausal women. Am J Physiol Endocrinol Metab 2018;314:E448-56.

5. Thaung Zaw JJ, Howe PRC, Wong RHX. Postmenopausal health interventions:Time to move on from the Women's Health Initiative? Ageing Res Rev 2018;48:79-86.

6. Stefanska A, Bergmann K, Sypniewska G. Metabolic Syndrome and Menopause: Pathophysiology, Clinical and Diagnostic Significance. Adv Clin Chem 2015;72:1-75.

7. Schenck-Gustafsson K, Brincat M, Erel CT, et al. EMAS position statement: Managing the menopause in the context of coronary heart disease. Maturitas 2011;68:94-7.

8. Dennis MK, Burai R, Ramesh C, et al. In vivo effects of a GPR30 antagonist. Nat Chem Biol 2009;5:421-7.

9. Cao J, Mi Y, Shi C, et al. First-line anti-tuberculosis drugs induce hepatotoxicity: A novel mechanism based on a urinary metabolomics platform. Biochem Biophys Res Commun 2018;497:485-91.

10. Meyer MR, Clegg DJ, Prossnitz ER, et al. Obesity, insulin resistance and diabetes:sex differences and role of oestrogen receptors. Acta Physiol (Oxf) 2011;203:259-69.

11. Meyer MR, Fredette NC, Howard TA, et al. G proteincoupled estrogen receptor protects from atherosclerosis. Sci Rep 2014;4:7564.

12. Rosenfeld ME, Kauser K, Martin-Mcnulty B, et al. Estrogen inhibits the initiation of fatty streaks throughout the vasculature but does not inhibit intra-plaque hemorrhage and the progression of established lesions in apolipoprotein E deficient mice. Atherosclerosis 2002;164:251-9.

13. Kridawati A, Rahardjo TBW, Hardinsyah, et al. Comparing the effect of tempe flour and tofu flour consumption on estrogen serum in ovariectomized rats. Heliyon 2019;5:e01787.

14. Li M, Zeng $M$, Zhang ZG, et al. Lepidiumuridine A: A New Natural Uridine Derivative as a Phytoestrogen Isolated from the Seeds of Lepidium apetalum Willd. Evid Based Complement Alternat Med 2018;2018:2813465.

15. Cahyadi W. Soybean Benefits and Technology. Jakarta: Bumi Aksara, 2007.

16. Suciu CF, Prete M, Ruscitti P, et al. Oxidized low density lipoproteins: The bridge between atherosclerosis and autoimmunity. Possible implications in accelerated atherosclerosis and for immune intervention in 
autoimmune rheumatic disorders. Autoimmun Rev 2018;17:366-75.

17. Salvayre R, Negre-Salvayre A, Camare C. Oxidative theory of atherosclerosis and antioxidants. Biochimie 2016;125:281-96.

18. Barton M. Obesity and aging: determinants of endothelial cell dysfunction and atherosclerosis. Pflugers Arch 2010;460:825-37.

19. Barton M. Cholesterol and atherosclerosis: modulation by oestrogen. Curr Opin Lipidol 2013;24:214-20.

20. Bairey Merz CN, Johnson BD, Braunstein GD, et al. Phytoestrogens and lipoproteins in women. J Clin Endocrinol Metab 2006;91:2209-13.

21. Zhou J, Yoshitomi H, Liu T, et al. Isoquercitrin activates the AMP-activated protein kinase (AMPK) signal pathway in rat H4IIE cells. BMC Complement Altern Med 2014;14:42.

22. Berkoz M. Effect of Hyperoside on the Inhibition of Adipogenesis in 3t3-L1 Adipocytes. Acta Endocrinol (Buchar) 2019;15:165-72.

23. Tian X, Xiong Q, Chen L, et al. Intervention of Catalpol on High-fat Diet Induced Nonalcoholic Fatty Liver Disease in Mice. Zhongguo Yi Xue Ke Xue Yuan Xue Bao 2019;41:746-55.

24. Huang WC, Liao PC, Huang CH, et al. Osthole attenuates lipid accumulation, regulates the expression of inflammatory mediators, and increases antioxidants in FL83B cells. Biomed Pharmacother 2017;91:78-87.

25. Hwang YL, Im M, Lee MH, et al. Inhibitory effect of imperatorin on insulin-like growth factor-1-induced sebum production in human sebocytes cultured in vitro. Life Sci 2016;144:49-53.

26. Kwan HY, Wu J, Su T, et al. Schisandrin B regulates lipid metabolism in subcutaneous adipocytes. Sci Rep 2017;7:10266.

27. Yang Q, Wang C, Jin Y, et al. Disocin prevents postmenopausal atherosclerosis in ovariectomized LDLR/- mice through a PGC-1alpha/ERalpha pathway leading to promotion of autophagy and inhibition of oxidative stress, inflammation and apoptosis. Pharmacol Res 2019;148:104414.

28. Jing Y, Cai D, Chen Q, et al. Liuwei Dihuang soft capsules attenuates endothelial cell apoptosis to prevent atherosclerosis through GPR30-mediated regulation in ovariectomized ApoE-deficient mice. J Ethnopharmacol 2017;208:185-98.

29. Yang K, Zhang H, Luo Y, et al. Gypenoside XVII Prevents Atherosclerosis by Attenuating Endothelial Apoptosis and Oxidative Stress: Insight into the ERalpha-Mediated PI3K/Akt Pathway. Int J Mol Sci 2017;18:77.

30. Wing LY, Chen YC, Shih YY, et al. Effects of oral estrogen on aortic ROS-generating and -scavenging enzymes and atherosclerosis in apoE-deficient mice. Exp Biol Med (Maywood) 2009;234:1037-46.

31. Zhou C, Jia HM, Liu YT, et al. Metabolism of glycerophospholipid, bile acid and retinol is correlated with the early outcomes of autoimmune hepatitis. Mol Biosyst 2016;12:1574-85.

32. Herrero-Fernandez B, Gomez-Bris R, Somovilla-Crespo B, et al. Immunobiology of Atherosclerosis: A Complex Net of Interactions. Int J Mol Sci 2019;20:5293.

33. Von Eckardstein AE. Atherosclerosis: Diet and Drugs. Handbook of Experimental Pharmacology. SpringerVerlag Berlin-Heidelberg, 2005.

34. Abeywardena MY, Head RJ. Longchain n-3 polyunsaturated fatty acids and blood vessel function. Cardiovasc Res 2001;52:361-71.

35. Pfrommer CA, Erl W, Weber PC. Docosahexaenoic acid induces ciap $1 \mathrm{mRNA}$ and protects human endothelial cells from stress-induced apoptosis. Am J Physiol Heart Circ Physiol 2006;290:H2178-86.

36. Cotter DG, Schugar RC, Crawford PA. Ketone body metabolism and cardiovascular disease. Am J Physiol Heart Circ Physiol 2013;304:H1060-76.

37. Rojas-Morales P, Tapia E, Pedraza-Chaverri J. betaHydroxybutyrate: A signaling metabolite in starvation response? Cell Signal 2016;28:917-23.

38. Cheng B, Lu H, Bai B, et al. d-beta-Hydroxybutyrate inhibited the apoptosis of PC12 cells induced by $\mathrm{H} 2 \mathrm{O} 2$ via inhibiting oxidative stress. Neurochem Int 2013;62:620-5.

39. Mikami D, Kobayashi M, Uwada J, et al. betaHydroxybutyrate, a ketone body, reduces the cytotoxic effect of cisplatin via activation of HDAC5 in human renal cortical epithelial cells. Life Sci 2019;222:125-32.

40. Oni-Orisan A, Edin ML, Lee JA, et al. Cytochrome P450derived epoxyeicosatrienoic acids and coronary artery disease in humans: a targeted metabolomics study. J Lipid Res 2016;57:109-19.

41. Spiecker M, Liao JK. Vascular protective effects of cytochrome p450 epoxygenase-derived eicosanoids. Arch Biochem Biophys 2005;433:413-20.

42. Node K, Huo Y, Ruan X, et al. Anti-inflammatory properties of cytochrome P450 epoxygenase-derived eicosanoids. Science 1999;285:1276-9.

43. Zhou C, Huang J, Li Q, et al. CYP2J2-derived EETs attenuated ethanol-induced myocardial dysfunction 
through inducing autophagy and reducing apoptosis. Free Radic Biol Med 2018;117:168-79.

44. Magil SG, Zeisel SH, Wurtman RJ. Effects of ingesting soy or egg lecithins on serum choline, brain choline and brain acetylcholine. J Nutr 1981;111:166-70.

45. Wang X, Li N, Liu B, et al. A novel human phosphatidylethanolamine-binding protein resists tumor necrosis factor alpha-induced apoptosis by inhibiting mitogen-activated protein kinase pathway activation and phosphatidylethanolamine externalization. J Biol Chem 2004;279:45855-64.

46. Goumon Y, Angelone T, Schoentgen F, et al. The hippocampal cholinergic neurostimulating peptide, the N-terminal fragment of the secreted phosphatidylethanolamine-binding protein, possesses a new biological activity on cardiac physiology. J Biol Chem 2004;279:13054-64.

47. Kroslak T, Koch T, Kahl E, et al. Human phosphatidylethanolamine-binding protein facilitates heterotrimeric $\mathrm{G}$ protein-dependent signaling. J Biol Chem 2001;276:39772-8.

48. Gay I, Schwartz Z, Sylvia VL, et al. Lysophospholipid regulates release and activation of latent TGF-beta1 from chondrocyte extracellular matrix. Biochim Biophys Acta 2004;1684:18-28.

49. Wymann MP, Schneiter R. Lipid signalling in disease. Nat Rev Mol Cell Biol 2008;9:162-76.

50. Yamatani H, Takahashi K, Yoshida T, et al. Differences in the fatty acid metabolism of visceral adipose tissue in postmenopausal women. Menopause 2014;21:170-6.

Cite this article as: Huang $\mathrm{D}$, Hu H, Chang L, Liu S, Liang J, Song Y, Wang X, Zhang H, Wei C, Wu Y. Chinese medicine Bazi Bushen capsule improves lipid metabolism in ovariectomized female ApoE ${ }^{-/-}$mice. Ann Palliat Med 2020;9(3):1073-1083. doi: 10.21037/apm-20-906 